\title{
Der Maler Robert Sterl und sein Garten in Naundorf
}

\author{
Andreas Quermann
}

$\mathrm{B}$ ei Erstellung dieses Vortrages machte mich Frau Kristina Popova, die Verfasserin des Werkverzeichnisses Robert Sterls, darauf aufmerksam, dass wir in diesem Jahr den 100. Jahrestag des Sterl-Haus-Gartens feiern: Das im Stil der sogenannten Heimatschutzarchitektur errichtete Wohnhaus wurde 1912/13 für das Ehepaar Sebastian erbaut und anschließend bis 1914 zusammen mit dem umliegenden Gelände zu einem familiären Wohnort gestaltet. Obwohl der Dresdner Maler und Akademieprofessor Robert Sterl Haus und Grundstück erst nach dem Weltkrieg 1919 von der verwitweten Frau Sebastian erwarb, ein Atelier anbaute und dann zwölf Jahre bis zu seinem Tod 1932 dort lebte und arbeitete, so stammt die Grundstruktur des Hauses, des Gartens und Geländes im Wesentlichen noch aus der Erbauungszeit des Hauses vor einhundert Jahren: ein unterkellertes, zweigeschossiges, reich mit Fenstern durchlichtetes Landhaus mit spitzem Satteldach, Giebeln und Erker (Abb. 1).

\section{Robert Sterl}

Wer sich für die Kunstgeschichte Dresdens interessiert oder für die Malerei des deutschen Impressionismus, der kennt sicher den Maler Robert Sterl - dennoch ein paar allgemeine Worte zu seiner Biografie:

Sterl (Abb. 2) wurde 1867 in Großdobritz, heute Dresden-Dobritz, geboren. Er studierte bis 1888 an der Dresdner Kunstakademie Malerei. Nach seinem Studium war er um 1900 einige Jahre als freischaffender Landschaftsmaler und Porträtist tätig. Er war Mitglied im Kreis der Goppelner
Malergruppe um Carl Bantzer (1857-1941) und Wilhelm Claudius (1854-1942). Zum Broterwerb führte er eine private Damenschule und nahm Illustrationsaufträge an. Einer dieser Aufträge führte ihn 1894 in die Steinbrüche der Sächsischen Schweiz und daher rührt auch eines seiner Hauptthemen: $\mathrm{Zu}$ seinen bekanntesten Motiven zählen die Steinbrecher und Sandsteinbrüche der Sächsischen Schweiz. 1906 erfolgte die Berufung als Professor an die Dresdner Kunstakademie, wo Sterl bis 1931 lehrte. Er nahm in dieser Zeit entscheidenden Einfluss auf das Dresdner Kunstleben.

\section{Zum Vortrag}

Mein Vortrag fußt auf einer ersten Sichtung bisher nicht systematisch ausgewerteter Unterlagen und Dokumente in Sterls Nachlass. Ich biete Ihnen somit eine vorläufige Zustandsbeschreibung und fragmentarische Materialsammlung zum Garten und Grab, denn als Museumsleiter war ich zuvor weniger mit Gartengeschichte oder -gestaltung befasst, als mit praktischen Fragen - wie zum Beispiel mit der Überlegung, welche Bäume gefällt werden müssen, da sie Strommasten gefährden könnten; oder mit dem "Besuch « eines Autofahrers, der im vergangenen Winter unseren Gartenzaun beschädigte oder wie aktuell mit akuter Erosion der Kreisstraße und dauerhafter Schädigung des umgebenden Zaunes nahe der SterlGrabstätte.

Einige Dokumente kann ich erstmals öffentlich machen und darf zudem einige Fragen aufwerfen, wie man in Zukunft mit dem Garten umgehen sollte, welche Ein- 


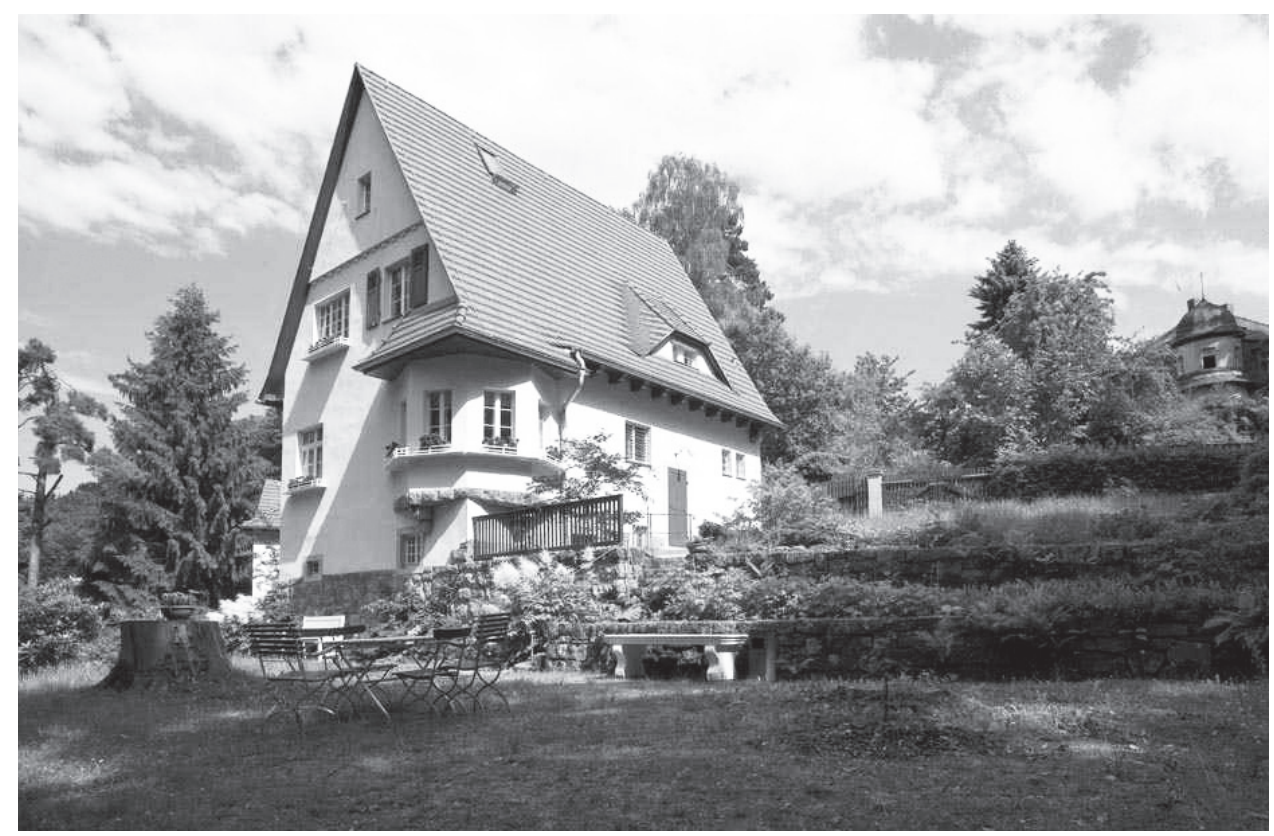

1 | Haus und Garten Robert Sterls, Fotografie: Herbert Boswank (Robert-Sterl-Haus).

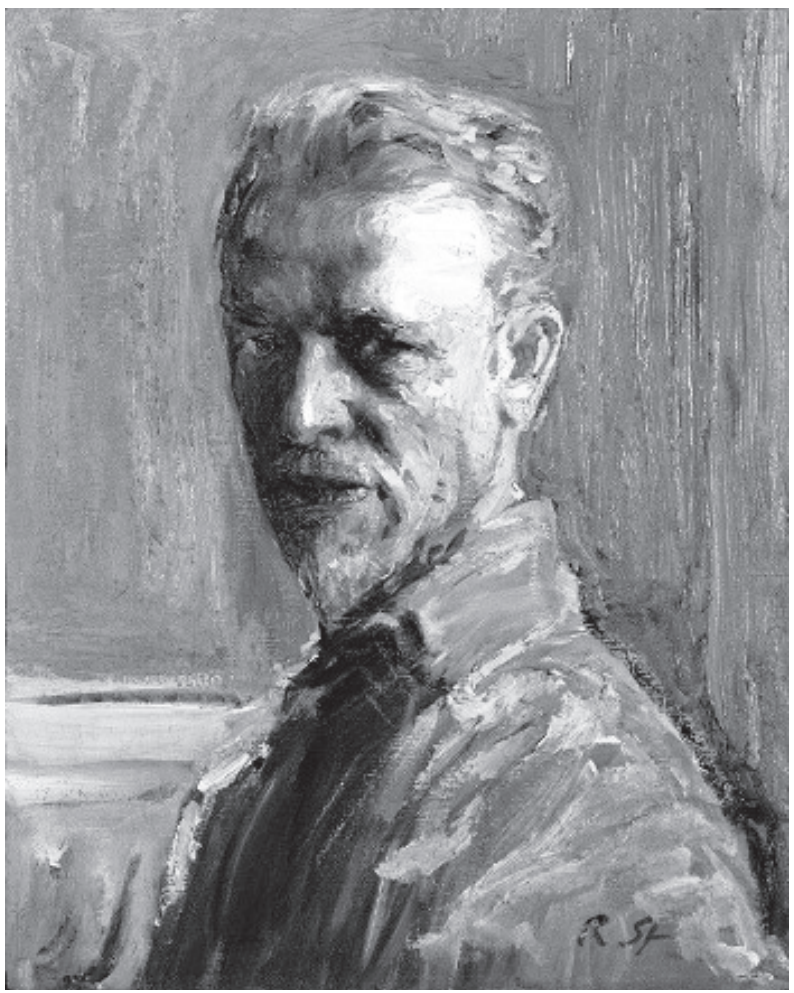

2 | Selbstbildnis Robert Sterls, 1920, WVZ 1077 (Robert-Sterl-Haus). 
griffe und Projekte erlaubt, sinnvoll und vor allem realisierbar - d.h. finanzierbar erscheinen. Doch erlauben Sie mir als Kunsthistoriker, dass ich mich dem Garten und der darin liegenden Grabstätte zunächst auf zwei Umwegen nähere.

\section{Kunsthistorische Annäherung}

Dazu möchte ich zu Beginn auf eine viel versprechende, aber irreführende Konstellation hinweisen: Ein bedeutender Maler, Impressionist sogar, also auch Landschaftsmaler und sein Garten! Da wird man hellhörig: Viele denken sofort an den Garten von Max Liebermann (1847-1935), an dessen Villa am Berliner Wannsee. Diesen prachtvollen Garten sieht man auf zahlreichen Gemälden des Meisters - welche Blütenpracht, welch Farbexplosion! In der Kunstgeschichte hat sich aber vor allem ein anderer Garten in das kollektive Bildgedächtnis eingebrannt: Claude Monets (1840-1926) Seerosenteich ist weltberühmt. Auch der Begründer des französischen Impressionismus war Landschaftsmaler und Gartenbesitzer, der sich wie Max Liebermann in seinem Spätwerk intensiv dem eigenen Garten widmete.

Wie anders dagegen der Umgang des sächsischen Impressionisten mit seinem Garten in Naundorf! Gemälde, die diesen Garten zeigen, müssen schon gesucht werden. Bekannt ist nur ein einziges, ausgestellt im Atelier des Robert-Sterl-Hauses: "Der Kirschzweig « vom 24.05.1920 - ein Detail, farbkräftig durchgestaltet mit komplementären Farben in Grün-Rot vor leuchtendem Himmelsblau. Die Komposition wird durch Diagonalen geprägt. Vom Garten ist jedoch nichts zu sehen.

Lediglich in einer Zeichnung gibt Robert Sterl eine Impression von der Lage seines Hauses im Garten: Halb geduckt liegt es hinter dem Hang, im Vordergrund stehen Obstbäume. Was aussieht wie Schnee, ist die »Kirschblüte in Naundorf» im April 1920 (Abb. 3). ${ }^{2}$

\section{Zur geografischen Lage}

Das Robert-Sterl-Haus mit seinem Garten liegt im Struppener Ortsteil Naundorf im Landkreis Sächsische Schweiz-Osterzgebirge - etwa $30 \mathrm{~km}$ östlich von Dresden bzw. $10 \mathrm{~km}$ von Pirna entfernt und für Wanderer günstig am beliebten Malerweg sowie in der Nähe des Elberadweges. Der Eingang zum Grundstück und zum Haus befindet sich an der Kreisstraße nach Naundorf, die heute den Namen Robert Sterls trägt, Hausnummer 30 - zu Sterls Zeit lautete die Adresse noch Naundorf Nr. 40, Post: Pötzscha.

Ein Teil des Geländes zieht sich zur Elbe hin hangabwärts bis zum Wehlener Ortsteil Pötzscha. Hier liegt auch der S-Bahnhof Stadt Wehlen, der allen Gästen des Museums als Anreiseweg empfohlen wird, nicht nur aus Gründen des Umweltschutzes, sondern auch, weil Robert Sterl selber täglich diesen Weg nach Dresden zu seiner Arbeit als Professor an der Dresdner Kunstakademie nahm.

In einem Skizzenbuch findet sich eine flüchtig skizzierte Wegbeschreibung (Abb. 4), ${ }^{3}$ die hier erstmals der Öffentlichkeit vorgestellt werden kann: Sterl deutet die Elbe mit der Elbfähre an. Die Lage der Stadt Wehlen auf der anderen Elbseite ist bezeichnet; diesseits liegt der Bahnhof, von wo die Straße nach Naundorf Schwung nimmt; oben liegt das Sterl-Haus. Am deutlichsten eingezeichnet ist der sich in Serpentinen den Elbhang emporschlängelnde Pfad auf dem eigenen Grundstück, den Sterl täglich zu nehmen pflegte und der heute nicht mehr öffentlich gangbar ist, aber von den Mitarbeitern des Museums als Abkürzung zum Bahnhof genutzt wird.

Das Grundstück liegt im seit 1956 ausgewiesenen Landschaftsschutzgebiet Sächsische Schweiz. Durch nachhaltige Nutzung der im Schutzgebiet liegenden Flächen soll sich die Landschaft von menschlichen Eingriffen wie dem Sandsteinabbau um die Jahrhundertwende erholen. Eine touristische Nutzung ist nur in geringem Maße gewünscht, der Siedlungsbau ist auf den 


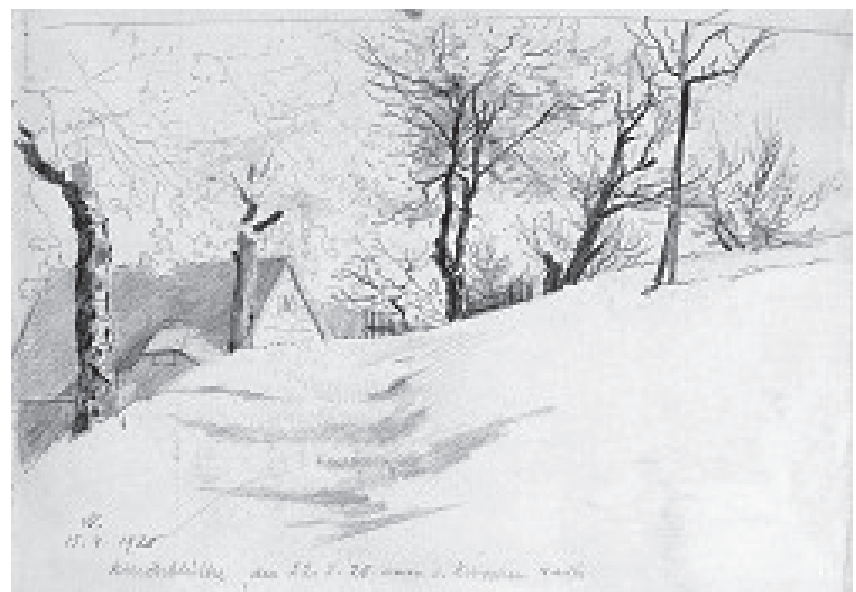

3 | »Kirschblüte in Naundorf «, Robert Sterl 1920, Bleistift auf Papier, Z 2211 (Robert-Sterl-Haus).

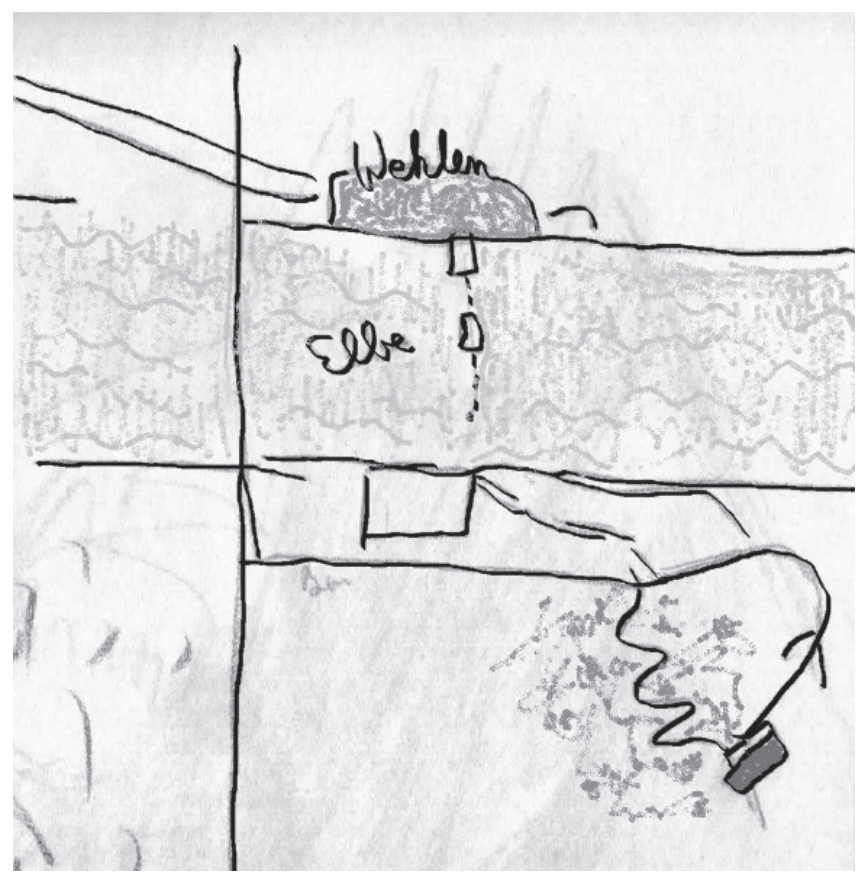

4 | Skizzierte Wegbeschreibung, Robert Sterl (undat.), Bleistift auf Papier, SKB 154-49 (Robert-Sterl-Haus). 
Eigenbedarf begrenzt. Die ansässigen Landund Forstwirtschaften werden dazu angehalten, nachhaltig zu wirken und zu produzieren. Und so wird auch der Garten des Sterl-Hauses ebenso nachhaltig gepflegt und nur in Teilen behutsam gestaltet.

\section{Das Grundstück}

Das Grundstück setzt sich zusammen aus den an der oberen Straße gelegene Naundorfer Flurstücken 377 und 378, wo das Haus steht, und dem schmalen, zur Elbe hin stark abfallenden Flurstück 103, das schon zur Gemarkung Wehlen-Pötzscha gehört. Laut einer Abschrift aus den Akten im Grundbuchamt vom 08.07.1957 in den Unterlagen der Sterl-Stiftung umfasste der Grundbesitz aus dem Nachlass der Witwe Robert Sterls insgesamt 1,35 Hektar - davon entfallen auf das eigentliche Haus samt Hofflächen und Wege nur umgerechnet $1.100 \mathrm{~m}^{2}$, wohingegen allein die als Wald ausgewiesene Fläche mit $7.100 \mathrm{~m}^{2}$ und die landwirtschaftliche Nutzfläche mit $5.300 \mathrm{~m}^{2}$ zu Buche schlägt.

Die landwirtschaftliche Nutzfläche teilte sich damals auf in $700 \mathrm{~m}^{2}$ Gartenland, wohl im unmittelbaren Bereich des Hauses, wo sich heute im Wesentlichen Wiesen und terrassierte Staudenbeete befinden. Dann gab es $1.700 \mathrm{~m}^{2}$ mit Obstanlagen, von denen nur wenige Reste mit alten und kaum mehr ertragreichen Apfelbäumen erhalten sind. Ein Kirschbaum wurde vor wenigen Jahren als Reminiszenz an die zuvor gezeigte SterlZeichnung neu gepflanzt. Die $1957 \mathrm{mit}$ $2.900 \mathrm{~m}^{2}$ genannte Viehweide existiert im Bogen der Straße nach Naundorf auf stark abfallendem Gelände noch unverändert.

Auf dem zur Elbe hin abfallenden Gelände, dem Flurgrundstück 103, wächst heute ein Mischwald ohne Unterholz mit mehr als vierzehn Eichen (bis zu $220 \mathrm{~cm}$ Stammumfang), über zwölf Birken (bis zu $140 \mathrm{~cm}$ ), etwa acht Hainbuchen (bis zu 150 $\mathrm{cm}$ ) und wenigen Kiefern. Interessant ist das über den Stammumfang ungefähr schätzbare Alter der Bäume, das allerdings nur durch eine Kernbohrung verifiziert werden könnte: Die Eichen kämen rechnerisch auf ein durchschnittliches Alter von etwa 170 Jahren, die Buchen dürften 90 Jahre alt sein, die Birken wesentlich jünger. Die Eichen standen somit schon, als Sterl das Haus 1919, also vor 95 Jahren erwarb, während die Buchen wohl erst in dieser Zeit anfingen zu wachen, jedenfalls schon bei Sterls Tod 1932, vor 82 Jahren, standen. Es ist anzunehmen, dass diese Bäume nicht bewusst angepflanzt wurden, sondern sich selbständig ausgesät haben.

Was bedeutet dieser Befund für den Umgang mit dem Waldgelände in Zukunft? Dazu zunächst eine andere Frage: Warum erwirbt jemand - in diesem Fall das Ehepaar Sebastian und danach Sterl - ein großes, abschüssiges Grundstück, das sich kaum landwirtschaftlich nutzen oder gar bebauen lässt? Eine allzu profane Antwort wäre: Gewinn von Brennholz. Schauen wir uns aber die Lage des Hauses auf dem Grundstück genauer an, so drängt sich die Vermutung auf, die Hausbesitzer wollten sich von ihrem Wohnzimmer und der Terrasse aus nicht den spektakulären Blick auf den Fluss verbauen oder zuwachsen lassen. Der Erwerb des Grundstückes in der schmalen Sichtachse zur Elbe hin ist somit als eine Art Versicherung für den freien Blick zu werten.

Hieraus ergibt sich für das Sterl-Haus ein Interessenskonflikt mit dem Landschaftsschutz: Erhalt der naturnahen Bewaldung oder Sichtschneise? Man hat sich für einen Kompromiss entschieden, der durchaus zu befürworten ist: Das Waldgelände wurde stark ausgedünnt, das Unterholz wird klein gehalten, aber die älteren Bäume mit hohen Stämmen bleiben stehen, sodass in den Baumkronen zwar ein dichter Wald gegeben ist, der sich jedoch im unteren Bereich lichtet. Durch das stark abfallende Gelände ist über Büsche hinweg ein Blick auf die Elbe und auf die gegenüber liegende Stadt Wehlen möglich.

Aus diesem Grund wurden vor einigen Jahren auch an der nördlichen Hausseite, zur Elbe hin, am oberen Ende des Flurstücks 


\section{Der Maler Robert Sterl und sein Garten in Naundorf}

103, mehrere große Blautannen entfernt und stattdessen ein pittoresker Rhododendron- und Azaleen-Garten angelegt. Diese blühende Farbenpracht in Weiß, Flieder, Rot, Gelb und Orange ist so gewählt, dass die verschiedenfarbenen Blüten über einige Wochen hinweg zu unterschiedlichen Zeiten zur Entfaltung kommen - zu erleben sind sie vor allem am Beginn jeder Saison im Mai.

Um trotz des Eingriffs in die Natur Lebensraum für heimische Tiere zu erhalten, wurden Schnittholz-Stapel und Benjeshecken angelegt. Dieses locker gelagerte Totholz bietet zahlreichen Vogelarten, Kleinsäugern und Insekten Lebensraum. In den Bäumen finden sich in geraumer Höhe hölzerne Nistkästen für verschiedene Vogelarten. Hieraus ergibt sich für die nähere Zukunft die Aufgabe, gezielt neue Nistkästen für besonders schützenswerte Arten zu platzieren. Auch ein sogenanntes »Insektenhotel « für den Garten wäre ggf. interessant.

Die bisher beschriebenen Baumfällungen und Eingriffe in den natürlichen Wuchs betrafen nur die unmittelbare Umgebung des Hauses und das schmale Flurstück 103.

Ein anderer Bereich des Grundstücks blieb dagegen bis heute unberührt: eine "schluchtartige Geländevertiefung «. Hier ist die Natur sich selbst überlassen und hat ein schwer $\mathrm{zu}$ durchdringendes Dickicht geschaffen, das nur im Winter überhaupt einsehbar ist. Wo genau die Grundstücksgrenze zum unbewohnten Nachbargrundstück mit der leer stehenden Villa Zauberblick verläuft, ist im Gelände nicht genau ersichtlich und ohne praktische Bedeutung, da dieser Bereich wohl seit Jahren nicht mehr betreten wurde.

Kein Wunder daher, dass immer wieder größere Wildtiere den Sterl-Haus-Garten passieren: An einer Stelle im Lattenzaun war jüngst ein Holz zerbrochen, links und rechts davon Schlammspuren, die auf Wildschweine schließen lassen, die allerdings lange nicht mehr gesichtet wurden. Öfter dagegen scheucht man Rehwild auf, das mit Vorliebe die Bepflanzung am Grab reduziert, weshalb z.B. Rosenbeete im Garten nur direkt am Eingang zur Straße hin realisierbar sind - wo selbst der geringe Straßenverkehr die Tiere fern hält. Blindschleichen und Eichhörnchen sind dagegen unspektakuläre Bewohner unseres Gartens, ganz zu schweigen von vereinzelten Pfifferlingen, Steinpilzen und einer größeren Waldmeisterwiese im Frühjahr.

Das Grab Robert Sterls im oberen Bereich des Grundstücks, ca. 30 m westlich des Hauses nahe der Straße nach Struppen, ist von großen Rhododendronbüschen umgeben und von der nahen Straße kaum sichtbar. Es wird in einigem Abstand von drei mächtigen Eichen umgeben, die mit einem Stammumfang von bis zu drei Metern auf ein Alter von etwa 120 bis 240 Jahren schließen lassen. Auf der anderen Seite, östlich des Hauses unterhalb des Obstgartens, stehen vier annähernd 100-jährige Ahorn-Bäume mit einem durchschnittlichen Stammumfang von $180 \mathrm{~cm}$. Dort ragt auch eine riesige Fichte von zwei Metern Umfang weit über das Dach des Atelieranbaues empor. Da Sterl das Haus vor 82 Jahren erwarb, müssen diese Bäume damals schon gestanden haben.

Gefällt wurden in den vergangenen Jahrzehnten - jeweils in Absprache und nach Genehmigung durch das zuständige Forstamt - drei große Bäume in unmittelbarer Nähe des Hauses: Die große Linde vorne, an der Straßenseite neben dem Hauseingang, musste 2009 weichen, da ihre Wurzeln das Fundament und die Mauern des Hauses sowie die Abwasserleitungen zerstört hatten. Am Hang hinter dem Haus wurden zwei Buchen aus Altersgründen gefällt, die schon Krankheiten zeigten und Besucher gefährdeten. Hier lässt der Umfang von $280 \mathrm{~cm}$ des einen verbliebenen Stumpfes auf ein Alter von etwa 170 Jahren schließen.

Was lässt sich nun aber über gestalterische Eingriffe durch den Künstler sagen? - Die Dokumente geben nichts her. Es liegen Rechnungen über Umbaumaßnahmen und Sanierungen am Haus vor, auch über eine neue Dampfheizung oder die Anschaffung eines Radios. Zum Garten wissen wir derzeit 
nur, dass ein Hundezwinger angelegt und des Öfteren Maschendrahtzaun erworben wurde, was nicht auf eine ambitionierte Gestaltungsfreude hindeutet. Und während zahlreiche Hundezeichnungen existieren, lässt sich kaum eine Landschaftsdarstellung des Künstlers seinem Garten zuordnen.

\section{Zum Grab}

Regelmäßig fragen Besucher und Gäste des Museums nach dem Grabmal und wie es bloß möglich gewesen sei, im eigenen Garten beerdigt zu werden. - Wie kam es also dazu?

Auf eigenen Wunsch wurde der Leipziger Grafiker, Maler und Bildhauer Max Klinger (1857-1920) am 08.07.1920 auf seinem Weinberg in Großjena bei Naumburg beerdigt. Die Gestaltung der Grabstätte erfolgte durch Johannes Hartmann, einen Freund Klingers. Über dem Grab wurde nach Vorgabe des Verstorbenen eine Bronze-Figur aufgestellt. Den Zugang zur Grabstätte säumen zwei Marmor-Hermen, die Klinger und seine Frau Gertrud zeigen. Am 16.07.1920 berichtete das Naumburger Tageblatt ausführlich über die feierliche Beerdigung Klingers. Als einer der Honoratioren wird auch erwähnt: »Für den Akademischen Rat in Dresden spricht Professor Sterl«.

Wahrscheinlich kam Sterl hier die Idee, nach dem Vorbild Klingers selber für eine letzte Ruhestätte vorzusorgen, denn das Ehepaar Sterl war kinderlos geblieben. Es hatte somit keine Erben und musste den Verbleib des Vermögens, des Hauses mit Grundstück und der im Nachlass befindlichen Werke regeln. Das Testament der Eheleute Sterl legte die Gründung einer Stiftung zur Förderung junger Kunststudenten der Dresdner Akademie fest, um damit auch das Andenken an den in der Lehre überaus engagierten Akademieprofessor zu bewahren.

Schon von Krankheit gezeichnet, hatte Sterl im Januar 1931 die Bestattung auf dem eigenen Grundstück in Naundorf offiziell beantragt. Ein behördliches Antwortschrei ben des Amtshauptmanns vom 26.01.1931 empfiehlt das weitere Vorgehen im Genehmigungsverfahren: "Zur Errichtung eines Erbbegräbnisses außerhalb des Friedhofes bedarf es der Genehmigung des Ev.-luth. Landeskonsistoriums in Dresden. Zuvor ist der Bezirksarzt zu hören. Wenn beabsichtigt ist, wie ich annehme, eine Baulichkeit zu errichten, bedarf es außerdem noch der Genehmigung der Amtshauptmannschaft als Baupolizeibehörde. Ich gebe daher anheim und möchte dazu raten, zwei getrennte Gesuche mit Lageplan und Bauzeichnungen zu richten 1.) an das Bezirkskirchenamt Pirna, damit dieses nach Gehör des Bezirksarztes die Sache an das Konsistorium einberichtet und 2.) an die Amtshauptmannschaft Pirna, Bauabteilung. Im übrigen darf ich der Hoffnung Ausdruck geben, (...) daß die ganze Angelegenheit zwar vorbereitet, aber noch lange lange Jahre nicht praktische Bedeutung bekommen werde."

In einem weiteren Schreiben wenige Tage später wird dann am 07.02.1931 korrigiert, »dass es im vorliegenden Falle einer besonderen Baugenehmigung nicht bedarf. Voraussetzung ist allerdings, dass die Gruft mindestens $4 \mathrm{~m}$ von der Grenze entfernt ist, damit durch die Abgrabungen das Nachbargrundstück nicht etwa beeinträchtigt wird."

Schließlich schickt der Bezirksarzt am 05.03.1931 in der Angelegenheit seinen Bericht an das Bezirkskirchenamt. Daraus ist eine längere Passage zu zitieren, da die genaue Beschreibung der Topografie bis heute zutrifft: "Das Gesuch des Professor Sterl auf Anlegung einer Grabstelle für 2 Personen auf eigenem Grund und Boden, der nach dem Tode in Staatsbesitze übergehen soll, ist von mir (...) in medizinalpolizeilicher Hinsicht geprüft worden. / Als Grabstätten kommen zwei Stellen in Frage, die nur $10 \mathrm{~m}$ voneinander entfernt sind, die eine »an den 4 Eichen « und eine andere »am Wege« kurz vorher.« Weiter heißt es, die Grabstelle sei genügend weit vom Haus und Nachbargrundstücken entfernt. Sie könne von dort auch nicht gesehen werden, da sie "von außen durch einen Bretterverschlag, 


\section{Der Maler Robert Sterl und sein Garten in Naundorf}

später auch durch Baumgruppen abgetrennt« sei, was heute tatsächlich der Fall ist. "Der Grund und Boden ist Lehmboden und es bestehen auch in dieser Beziehung keine Bedenken, an den genannten Stellen eine ausgemauerte Gruft für 2 Särge oder 2 nebeneinander gelegene ausgemauerte Gräber anzulegen.

Das Gelände senkt sich stark nach einer schluchtartigen Geländevertiefung, die aber keinen Bachlauf zeigt, überhaupt nur bei besonders starken Niederschlägen Wasser führt und in deren Bereich bis zum Elblauf keine Wassergewinnungsanlage vorhanden ist. Außerdem ist die Gruft noch ca. 70 bis $80 \mathrm{~m}$ von dieser Talsole entfernt und dürfte kaum einen Einfluß darauf auszuüben vermögen. Die Gruft oder die Gräber sind gut auszumauern und vollkommen dicht (ohne Entlüftung) abzudecken.« Abschließend fasst der Bezirksarzt zusammen: »Da es sich um ein staatseigenes Grundstück handelt [was nicht korrekt ist], das in Zukunft seine Besitzer nicht wechseln dürfte [was tatsächlich zutrifft], da ferner die genannten Grabstellen in medizinalpolizeilicher Hinsicht als unbedenklich bezeichnet werden können, wird das Gesuch diesseits durchaus befürwortet «. ${ }^{4}$

Knapp ein Jahr später starb Robert Sterl am 10.01.1932 im Alter von 64 Jahren in seinem Haus in Naundorf. Die Totenmaske nahm der Bildhauer Karl Albiker ab. Robert Sterl wurde, wie geplant, auf seinem Naundorfer Grundstück bestattet.

Erst fünf Jahre nach seinem Tod konnte die Gestaltung des Grabmals im Auftrag der Witwe Helene Sterl durch den Dresdner Architekten Otto Rometsch (1878-1938) realisiert werden. Rometsch hatte in Karlsruhe bei Carl Schäfer (1844-1908) Architektur studiert, einem der wichtigsten Vertreter der späten Neugotik sowie einer der Vorläufer des Heimatschutzstils, der als Denkmalpfleger die Vollendung der Meißner Domtürme (1903-1908) verantwortete. In dieser Zeit, um 1906, war Rometsch nach Sachsen gezogen, zunächst nach Niederlößnitz. In Dresden widmete sich der Architekt, mit
Büro vertreten am Dresdner Altmarkt, als Gutachter und bei Restaurierungsarbeiten an zahlreichen sächsischen Schlössern und Kirchen vor allem der Denkmalpflege. Vielleicht ist dies ein Grund, warum sich die von ihm entworfene Grabstätte Sterls unter geschickter Nutzung des Geländeprofils so dezent und einfühlsam in die Gartenlandschaft einfügt. Anders als die vielgestaltige und dramatisch inszenierte Anlage des Klinger-Grabmals - was dessen künstlerischem Werk durchaus entspricht - ist das Sterl-Grab, dem Charakter seines Wesens entsprechend, eher zurückhaltend gestaltet.

Ein auf flachem Postament platzierter Sandsteinkubus mit den Maßen 65 x 125 x $100 \mathrm{~cm}$ (Höhe $\mathrm{x}$ Breite $\mathrm{x}$ Tiefe) trägt auf wiederum zweifach zurückgestuftem Sockel eine einfache, flache Sandsteinschale für Bepflanzungen - Gesamthöhe mit Schale: $130 \mathrm{~cm}$. Eine schlichte, gerade geführte Sandsteintreppe führt aus dem niedriger gelegenen Gartenbereich sieben Stufen hinauf zum Kubus mit der Inschrift: ROBERT STERL. Diese eigentliche Hauptansicht des Grabmals, dies wird aus den Plänen deutlich, wird heute kaum noch beachtet. Die meisten Besucher nähern sich seit Jahren direkt von der mit dem Haus ebenerdigen Seite, wo sich auch die neuere, wohl nach dem Tod der Witwe 1950 eingefügte Inschrift befindet:

HIER RUHEN DER MALER ROBERT STERL // PROFESSOR DER AKADEMIE DER BILDENDEN KUENSTE VON 1904 BIS 1932 // DOCTOR HONORIS CAUSA DER PHILOSOPHISCHEN FAKULTAET LEIPZIG // GEBOREN AM 23 JUNI 1867 GESTORBEN AM 10 JANUAR 1932 // UND FRAU HELENE STERL // GEBORENE HEDELT // GEBOREN AM 29 JANUAR 1873 GESTORBEN AM 11 NOVEMBER 1950.

Aufschlussreich ist ein Vergleich des realisierten Grabmals mit zwei Plänen des Architekten vom Mai 1937 (Abb. 5 und 6), 
AHA!

Miszellen zur Gartengeschichte und Gartendenkmalpflege
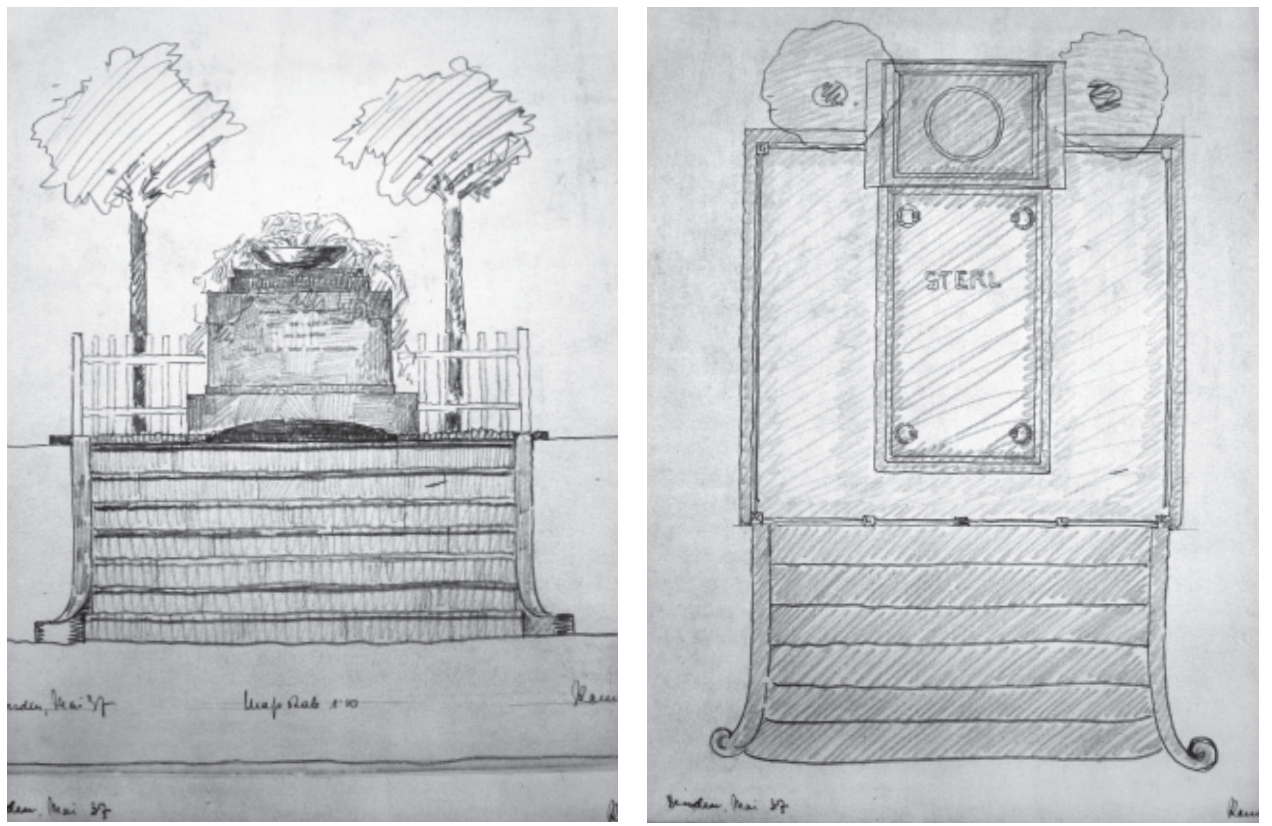

5 | 6 | Entwürfe zum Grabmal Robert Sterls, Otto Rometsch 1937 (Robert-Sterl-Haus).

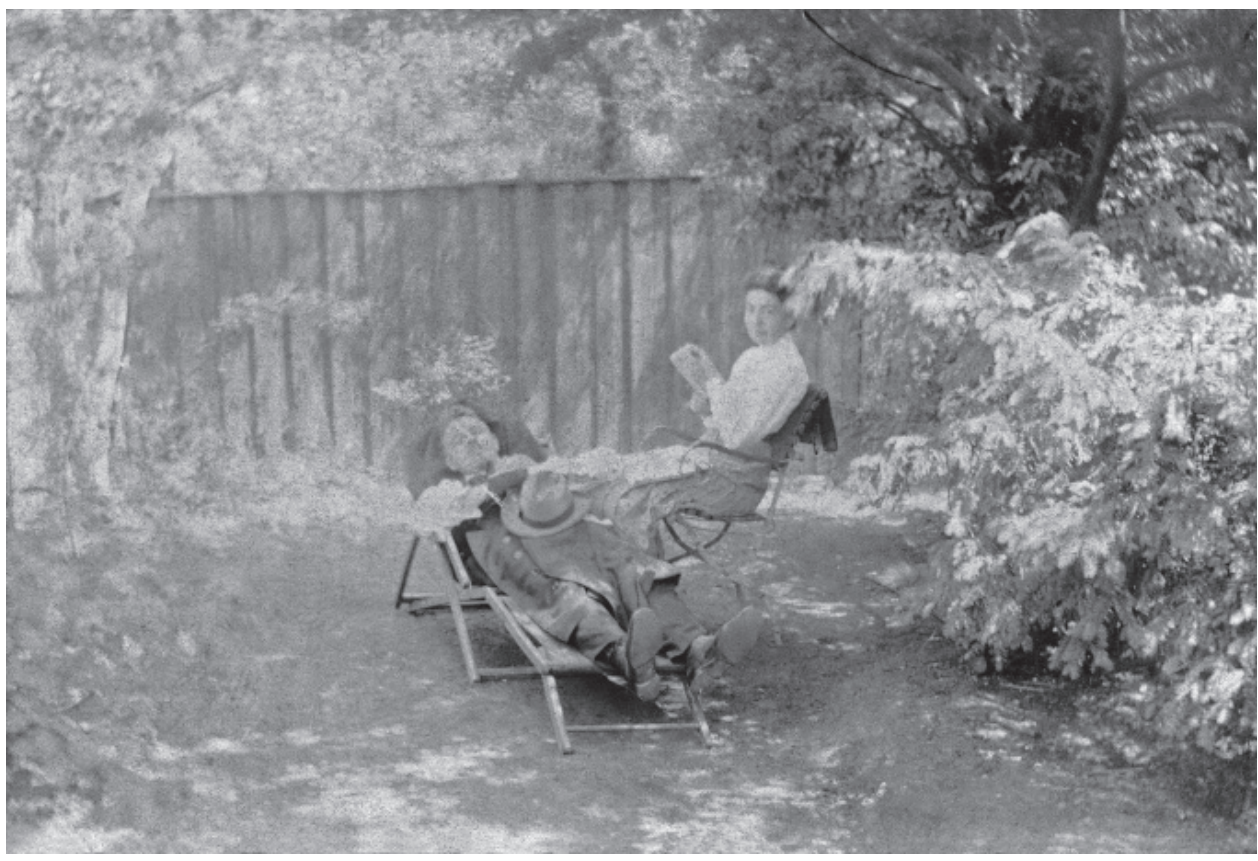

7 | Robert und Helene Sterl in ihrem Garten, unbekannter Fotograf (undat.) (Robert-Sterl-Haus). 


\section{Der Maler Robert Sterl und sein Garten in Naundorf}

die hier erstmals öffentlich gezeigt werden können: Das Grabmal wurde dem Plan entsprechend errichtet; nur die am unteren Lauf leicht ausschwingende Treppe erscheint vereinfacht und fällt wesentlich schmaler aus als im Entwurf vorgesehen. Die gezeichnete Grabplatte mit den Ringen ist nicht vorhanden, wohl aber der umlaufende Sandsteinsaum. Einen Zaun gibt es nicht, und auch die den Kubus flankierenden Birken sind nicht mehr da, müssen aber vor über zehn Jahren noch gestanden haben, wie sich langjährige Mitarbeiter erinnern. Eine Neuanpflanzung dieser Bäume wäre aus denkmalpflegerischen Gründen wünschenswert.

In der Kostenzusammenstellung vom 22.09.1937 berechnete Otto Rometsch für das Grabmal insgesamt 2.681 Reichsmark. Aus den oben zitierten Schreiben des Genehmigungsverfahrens war noch nicht hervorgegangen, ob »eine ausgemauerte Gruft» oder zwei "nebeneinander gelegene ausgemauerte Gräber« errichtet würden. In der Architektenrechnung wird nun aber eine "Gruftplatte" in Rechnung gestellt, sodass wir von einem Gruftbegräbnis ausgehen können. Pläne der unterirdischen Anlage sind in den Bauunterlagen nicht auffindbar. Auch wurde die Gruft seit der Bestattung Helene Sterls 1950 nie wieder geöffnet.

\section{Schluss}

Eine kleine, stark vergilbte Fotografie zeigt das Ehepaar Sterl in seinem Garten bei einem sonnigen Mußestündchen (Abb. 7): Der Maler liegt, mit Sonnenhut auf dem Bauch, entspannt in einem Liegestuhl, seine Ehefrau Helene sitzt daneben in einem Gartenstuhl. Im Hintergrund ist die damalige Begrenzung des Gartens durch einen Sichtschutz aus Holz zu erkennen, wie er immer noch bei einem Teil des Grundstücks beim Grab zur Straße hin existiert. Auch die Gartenmöbel sind zum Teil erhalten und weiterhin durch unsere Museumsgäste in Benutzung.

Robert Sterl hatte sich an zahlreichen
Ausstellungen in Dresden und Berlin, aber auch international mit großer Anerkennung beteiligt. $\mathrm{Zu}$ seinen Lebzeiten fand jedoch nur eine große Einzelausstellung statt und zwar 1928 in Chemnitz. Ein zeitgenössischer Zeitungsbericht dazu sei zum Schluss kurz zitiert: »Nach dieser Ausstellung« so hieß es damals, "wird er sich wieder in Naundorf in sein Märchenhäuschen mit der Linde am Eingang, den Blumen und den Erdbeeren im Garten zurückziehen. In die Stille, welche die Dinge reifen läßt.»

Das Museum ist geöffnet von Mai bis Oktober, Donnerstag bis Sonntag und an Feiertagen jeweils von 10 bis 17 Uhr. Bei Ausstellungseröffnungen und Veranstaltungen wird der Garten regelmäßig zum Ort des Zusammenkommens kunstbegeisterter Menschen. Besucher und vor allem Wandergruppen sind eingeladen, sich im Garten zu erholen und ihr Picknick zu verzehren.

\footnotetext{
Öl auf Leinwand, 55,5 x $50 \mathrm{~cm}$, WVZ 1079 Bleistift auf Papier, Robert-Sterl-Haus, Z 2211.

Bleistift auf Papier, Robert-Sterl-Haus, SKB 154-49, digital nachkoloriert.

4 Fotokopie des Entwurfs eines amtlichen Schreibens ohne Briefkopf und Unterschrift im Archiv des Sterl-Hauses.
} 